\title{
When Regenerative Medicine Faces the Challenges of Reproductive Medicine: A Review Study on Recent Advances in the Strategies for Derivation of Gametes from Stem Cells
}

This comprehensive review by Gil Juliá and Medrano on recent advances on in vitro gametogenesis provides clues for what is still missing and what are the future steps to take to produce male and female gametes in vitro from stem cells. Clearly, the way ahead is still long and difficult, but the recent successes in cryopreservation of mature oocytes and ovarian tissue, and the birth of the first macaque conceived with spermatozoa obtained after transplantation of prepubertal testicular tissue, give hope for fertility preservation of prepubertal cancer patients.

\section{Prof Elisabetta Baldi}

University of Florence, Italy
Authors:
María Gil Juliá, ${ }^{*}$ José V. Medrano²
1. IVI Foundation, Instituto De Investigación Sanitaria La Fe de Valencia, Valencia, Spain
2. Reproductive Medicine Unit, Instituto De Investigación Sanitaria La Fe de Valencia, Valencia, Spain
*Correspondence to jomepla@gmail.com

Disclosure: The authors have declared no conflicts of interest.

Received:

Accepted:

Keywords:

Citation:
13.04 .20

14.06.20

Derivation, differentiation, oogenesis, pluripotent stem cells, primordial germ cells (PGC), spermatogenesis.

EMJ Repro Health. 2020;6[1]:42-52

\section{Abstract}

The murine model has allowed for the replication of all developmental stages of the mammalian germline in vitro, from embryonic stem cells to epiblast cells, primordial germ cells, and finally into functional haploid gametes. However, because of interspecies differences between mice and humans, these results are yet to be replicated in our species. Reports on the use of stem cells as a source of gametes, retrieved from public scientific databases, were analysed and classified according to the animal model used, the stem cell source and type, the differentiation strategy, and its potential application. This review offers a comprehensive compilation of recent publications of key events in the derivation of germ cells and gametogenesis in vitro, in both mice and human models. Additionally, studies intending to replicate the different stages in human cells in vitro, in order to obtain cells with a phenotype akin to functional human gametes, are also depicted. The authors present options for deriving gametes from stem cells in vitro and different reproductive options for specific 
groups of patients. Lastly, the potential applications of in vitro human gametogenesis are evaluated as well as the main limitations of the techniques employed. Even though it appears that we are far from being able to obtain gametes from pluripotent stem cells in vitro as a viable reproductive option, its current academic and clinical implications are extremely promising.

\section{INTRODUCTION}

Gametes are highly specialised cells that allow the birth of new individuals and, with that, the continuation of a species. Gametogenesis is a carefully orchestrated process in which primordial germ cells (PGC), the precursors of gametes, undergo specification, epigenetic reprogramming, and differentiation into functional adult gametes: spermatozoa and oocytes., In this context, the use of stem cells to derive germ cells in vitro during the last decade has allowed us for the first time to study the development of germ cells. This has paved the way to understanding key events that may help develop new strategies to produce functional gametes for infertile patients, unable to produce them, and allow their genetic parenthood in the future.

The specification of the germline starts in the embryo with the derivation of PGC from epiblast cells and because this occurs during the implantation of the embryo, it is an inaccessible process for research in humans. ${ }^{3}$ Therefore, the mechanisms involved in this process have been studied in different animal models, primarily mice. ${ }^{4}$ Even though the common elements within the array of transcription factors that regulate the specification of human and mouse PGC have been described, significant differences call for the design of models for the development of the germline and in vitro gametogenesis from human cells (Figure 1).5,6 The acquisition of the so-called 'germline fate' is the result of events such as the activation of the BLIMPI/PRDM1 and PRDM14 network by bone morphogenetic protein 4 (BMP4) produced by extraembryonic tissues, ${ }^{7}$ the repression of the mesodermal fate, and re-establishment of pluripotency. This is pivotal to achieve epigenetic reprogramming, ${ }^{1,6}$ as well as the expression of germline-specific factors PRDM1/BLIMP1, TFAP2C or DAZL, and pluripotency factors OCT4 and NANOG. However, key differences between the murine model and the human reside in how these factors interact and how they execute their function. ${ }^{5}$ After their specification in the posterior region of the epiblast in response to the extraembryonic signals described above, PGC multiply and migrate towards the genital ridges as they reprogramme their epigenome, erasing almost all epigenetic marks in their DNA with the exception of some families of transposable elements to avoid the transmission of epigenetic mutations to the offspring and reset imprinting marks according to their sex. Following this, in a gonadal sexdependent phase, germ cells undergo changes in their morphology and epigenome as they enter cell cycle arrest in meiosis prophase I (in the case of human female germ cells or oogonia) or in a quiescent premeiotic state (in the case of human male germ cells or gonocytes). It is their interaction with the gonadal niche that determines the continuation of meiosis and the remethylation pattern that will be followed after birth in oogonia or immediately after sex determination in gonocytes. ${ }^{1-3}$

Although our understanding of the molecular mechanisms involved in the production of human gametes is increasing, this information is still limited. It is mandatory to understand the specific events responsible for the derivation of human PGCS (hPGC) in vivo to be able to obtain hPGClike cells (hPGCLC) from pluripotent stem cells in vitro $^{6}$ and exploit their clinical applications consciously and safely. This raises the need to develop in vitro gametogenesis models, not only to obtain immediate treatments for specific groups of patients, but also to perfect the newest protocols that are currently arising for their differentiation in the laboratory while ensuring that the end product is safe and guarantees success. The aim of this comprehensive review is to compile recent research publications on key events in the derivation of germ cells and the replication of gametogenesis in vitro in both mice and human models, assessing the limitations of these findings, and discussing their potential applications in reproductive medicine. 

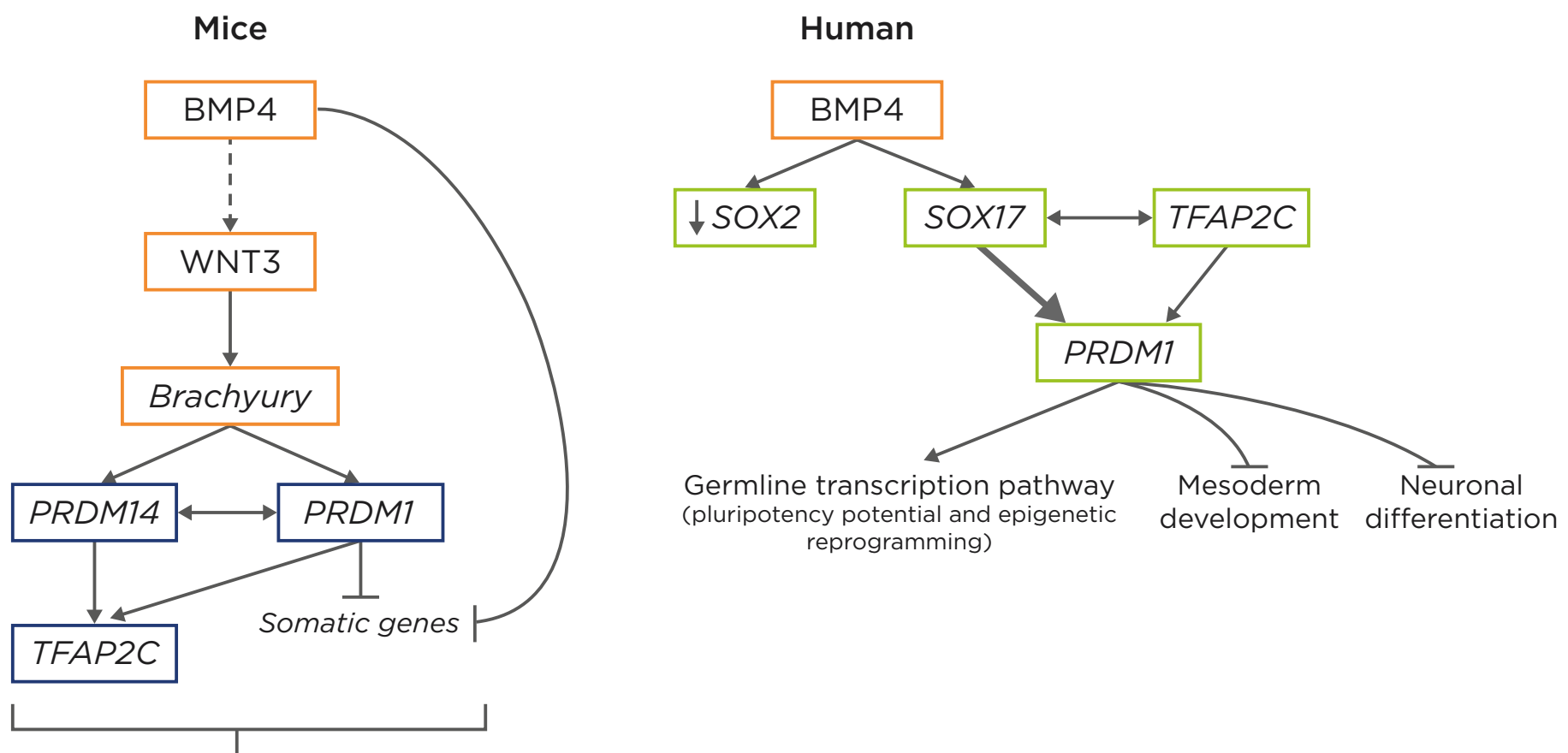

Germline transcriptional pathway

Sox2

Figure 1: Differences in molecular mechanisms involved in the specification of mice primordial germ cells versus human primordial germ cells in early post-implantation embryos. ${ }^{5,6}$

In mice, extra-embryonic ectoderm primordial germ cells (PGC)-competent cells secrete bone morphogenetic protein 4, which directly or indirectly activates the Wnt3 signalling pathway in the epiblastic cells, from which the activation of brachyury results, driving the first steps of differentiation during gastrulation. Brachyury activates both PRDM14 and PRDM1 inside PGC, driving the activation of TFAP2C and the inactivation of somatic genes, which trigger the expression of germline-specific transcription factors. Thus, the upregulation of PRDM1, PRDM14, and TFAP2C would suffice to induce a PGC-like state from mice epiblastic cells. In mice, SOX2 is a crucial pluripotency factor, which will recover its importance during mouse PGC specification later on. In humans, PGC-competent cells in the posterior epiblast have an increased expression of secrete bone morphogenetic protein 4, which will activate SOX17 inside the subset of cells connected to the mesoderm developing into human PGC. The upregulation of SOX17 is accompanied by the downregulation of the pluripotency marker SOX2, since their expression is mutually exclusive in humans, representing one of the main differences between mice and human early germline development. Also, brachyury, which was a main driver of mouse PGC, is not essential as an activator in humans. The network formed by SOX17, PRDM1, and TFAP2C is the main initiator of human PGC fate by activating the germline-specific transcriptomic pathway and inhibiting both the mesodermic and neuronal differentiation programmes.

Adapted from Kobayashi et al.5 and Saitou et al. ${ }^{6}$

\section{RECONSTRUCTION OF THE PRIMORDIAL GERM CELLS SPECIFICATION IN VITRO}

During the first decade of the 2000s, several reports focussed on the obtention of gametes in vitro from organotypic culture, either by maturation of fetal mice secondary ovarian follicles in culture from PGC to mature metaphase Il oocytes, ${ }^{8}$ or by retrieval of spermatogonial stem cells (SSC) from testicular biopsies. ${ }^{9}$ Despite the improvements in organ cell culture becoming a valuable tool for fertility treatments, this review focusses on the replication of the process of germline development from embryonic stem cells to the obtention of gametes.

To develop a model for the derivation of the germline in vitro, it is key to accurately replicate the transition between the two phases of pluripotency, naïve and primed, providing the starting pluripotent cells with competence to be specified into PGC first, and then completing 
their differentiation into PGC-like cells (PGCLC). Following this logic, Hayashi' ${ }^{10}$ et al. introduced a method to obtain mouse PGCLC (MPGCLC) from mice embryonic stem cells (mESC) in two steps. First they re-established the naïve pluripotent state of mESC using two kinase inhibitor cocktails and leukaemia inhibitor factor to start from a homogeneous pluripotent cell cohort ${ }^{1}$ that would mimic the properties of cells found in the internal mass of blastocysts, ${ }^{11}$ and pushed them to differentiate into epiblast-like cells (EpiLC) with competence to produce PGC. After that, using BMP4 plus a combination of other cytokines, the group achieved the differentiation of EpiLC into mPGCLC which were then transplanted into the mice testes to investigate if the cells were able to colonise the seminiferous tubules, complete meiosis, and mature. Once sperm were retrieved, they were used to fertilise oocytes by intracytoplasmic sperm injection (ICSI) and produced healthy offspring. This was the first evidence of the use of mice gametes obtained from pluripotent cells in vitro in assisted reproduction.1,10 One year later, the group used the same technique, this time replicating oogenesis and obtaining functional oocytes from aggregates of mPGCLC with somatic cells from mice embryonic ovaries. These aggregates were transplanted into the ovarian bursa, generating structures similar to follicles and oocytes within them. After ICSI using these retrieved oocytes, healthy offspring were obtained. Unfortunately, the method was proven to have limited efficiency, since a large subset of oocytes derived from mPGCLC were unable to extrude the second polar body after fertilisation, approximately $53 \%$ of the resulting zygotes were tripronuclear and, of those with two pronuclei, 35\% were dysgenic diploid zygotes, with two maternal pronuclei. Despite the aptitude for fertilisation of the resulting cells needing further investigation, these strategies set the foundation from which to attempt the reconstruction of the early stages of germline development in mammals. ${ }^{12}$

In humans, the two-step differentiation strategy has also been performed to obtain hPGC in vitro from human ESC (hESC) or human induced pluripotent stem cells (hiPSC). In 2015, Irie et al. cultured hESCwith a combination of 4 kinase inhibitors (4i) for the restoration of naïve pluripotency. EpiLC were also preinduced in response to BMP4 and derived into PGCLC, employing the same cytokine cocktail used by Hayashi et al. However, the authors noted that hPGCLC were able to derive directly from pluripotent cells in the $4 \mathrm{i}$ condition bypassing the preinduction state to EpiLC. Based on this, they concluded that the $4 \mathrm{i}$ state may be closer to the epiblast state than to a naïve pluripotent state, which explains why no preinduction was required..$^{13}$ In the same year, Sasaki et al. ${ }^{14}$ achieved similar results when using hiPSC from a non-naïve prepared pluripotent state, obtaining incipient mesodermal-like cells, which were differentiated following the beforementioned protocol into hPGCLC. ${ }^{14}$

From the differentiation strategies described, countless advances in the characterisation of the molecular mechanisms involved in the first step of the germline generation (until the obtention of PGCLC) have been made. The crucial characterisation of the network formed by factors PRDM1/BLIMP1, PRDM14 and TFAP2C in germinal fate specification in mice, ${ }^{15}$ the synergic action of SOX17 and PRDM1/BLIMP1, the progressive upregulation of NANOG and TFAP2C and downregulation of SOX2 in the human in vitro model, ${ }^{16}$ the role of TFAP2C in maintaining an open chromatin site in the naïve pluripotency enhancer OCT4 in hPGCs, ${ }^{4}$ the importance of the Wnt signalling pathway stimulation," and the dual behaviour of NANOG, PAX5, OCT4, and pRDM1 having more affinity to germline-specific genes in hPGCLC or pluripotency genes in hESC are a few examples of mechanisms involved in the early stages of gamete derivation (Figure 2). 4,13,16-18

\section{GAMETOGENESIS MODELS FOR THE IN VITRO OBTENTION OF GAMETES FROM PLURIPOTENT CELLS}

As a result of the differentiation protocols described above, other research groups have studied the mechanisms of the specification of PGC and their derivation into SSC), or oogonial stem cells to a deeper level.

\section{Spermatogenesis}

In 2016, Zhou et al..$^{19}$ applied the aforementioned Hayashi's two-step differentiation protocol to mESC and mice IPSC and obtained MPGCLC that were coaggregated with somatic testicular cells 
retrieved from mice carrying a mutation for c-kit, which produces a lack of endogenous germ cells. After the differentiation of the coaggregates in culture with follicle stimulating hormone, testosterone, bovine pituitary extract, BMP2 and BMP4, mPGCLC were able to progress through meiosis. Moreover, the haploid spermatid-like cells obtained presented the same imprint pattern as spermatids in vivo. Finally, spermatids were microinjected into oocytes, producing a healthy offspring. ${ }^{19}$

That same year, Ishikura et al. ${ }^{20}$ used Irie's two-step differentiation protocol, but in this case, mPGCLC were further isolated and cultured in suspension in coaggregation with somatic cells retrieved from fetal mice testis (Table 1). ${ }^{14,20-23}$ The majority of reconstituted testicular aggregates formed structures similar to seminiferous tubules with MPGCLC located within them. MPGCLC were subsequently differentiated into mouse germline stem cell-like cells (mGSCLC), which expressed pluripotency (tyrosine-protein kinase $\mathrm{KIT}$ ), germinal differentiation (DDX4, DAZL, SSEA1), and spermatogonial (PLZF) markers, resembling the phenotype of mouse germline stem cells (mGSC) in vivo. mGSCLC were then transplanted into germ cell-depleted adult mice testes.

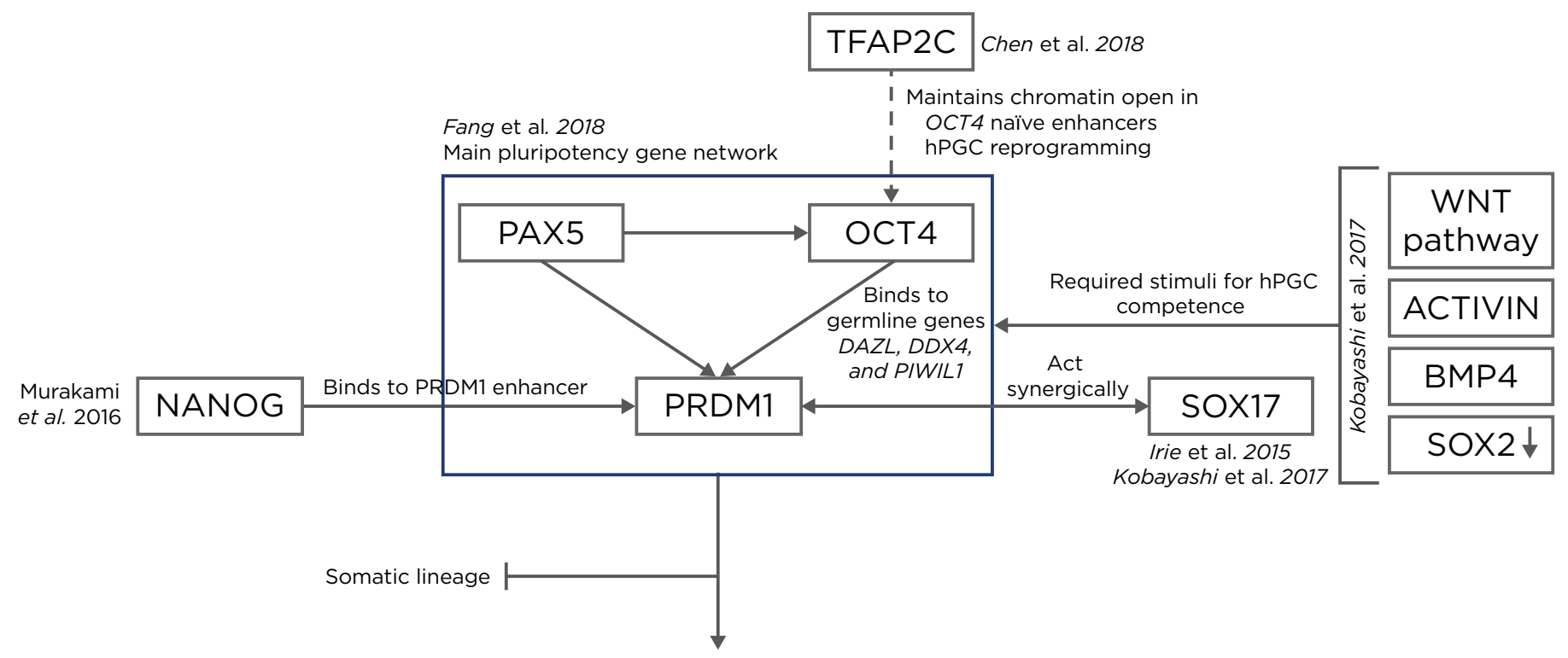

Primordial germ cell competence

Figure 2: Proposal for the characterisation of the molecular mechanisms of the early differentiation of human embryonic stem cells (hESC) into human primordial germ cells (hPGC). 4,13,16-18

SOX17 is key in the derivation of the human germline and the formation of the endoderm and it acts synergic with PRDM1 to confer pluripotent cells with primordial germ cell (PGC) competence, 13,16 together with stimuli from the Wnt signalling pathway, activin, bone morphogenic protein 4, and the downregulation of SOX $2 .^{16}$ Murakami et al. ${ }^{17}$ confirmed that NANOG was a key contributor to the germline fate of hESC while introducing that the role of that transcription could depend on the epigenetic context and stage. In mouse ESC, the role of NANOG is the maintenance of pluripotency, whereas in mouse PGC it binds to the enhancer region in PRDM1 to confer PGC competence. ${ }^{17}$ However, this study uses the mouse model and the suggested mechanism must be validated in human cells to be definitively included in the differentiation network from hESC to human PGC. Fang et al. ${ }^{18}$ studied the main pluripotency gene network in both hESC and hPGC. They suggested that PAX5 regulates the expression of OCT4 while they both act jointly over PRDM1 during PGC specification. The increase in expression of PRDM1 together with the binding affinity of OCT4 switching from pluripotent genes to germline-specific genes DAZL, DDX4 and PIWIL1, were characteristic of hPGC. ${ }^{18}$ In 2018 , Chen et al. ${ }^{4}$ noted that one of the roles of TFAP2C Is the opening of chromatin in naïve enhancers, one of them located in the OCT4 locus. The result is the induction of a brief second naïve pluripotency during which hPGC undergo epigenetic reprogramming. ${ }^{4}$ The result of all these interactions is the suppression of those genes that would deem the cells to the somatic lineage and, instead, the conferral of the PGC competence in order to develop the germline. 


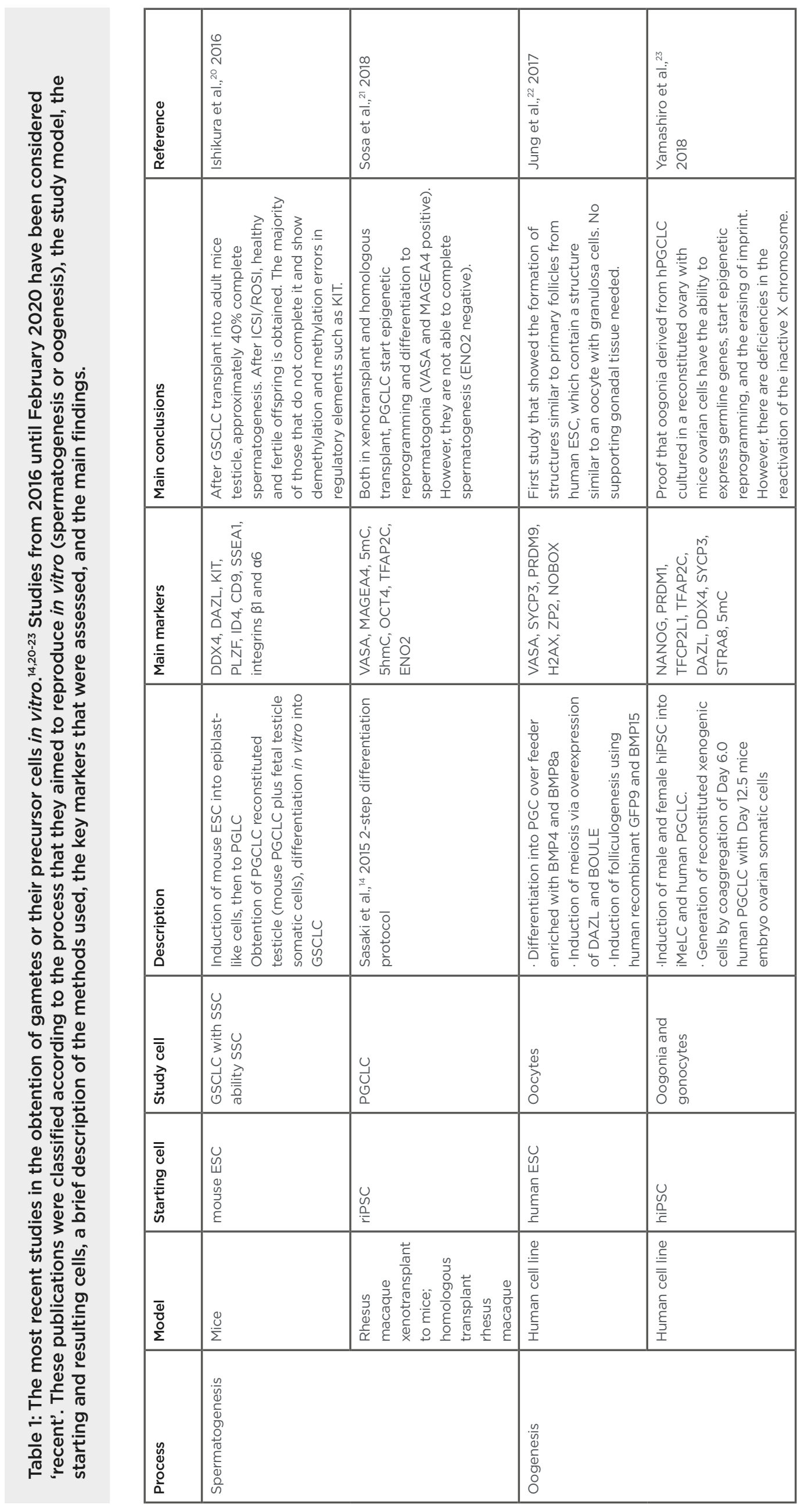

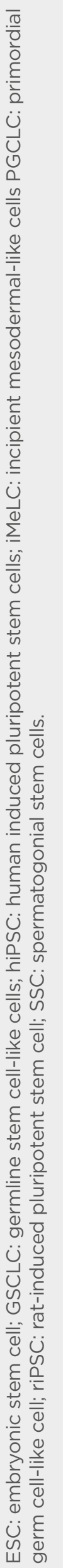


However, only around $40 \%$ of them were able to complete spermatogenesis and produce healthy offspring after microinjection into oocytes and because most mGSCLC stopped at meiosis prophase I, the methylation patterns in distinct stages of the differentiation were studied, unveiling errors in DNA methylation during mPGCLC differentiation. Therefore, even though the ability of mGSCLC to complete spermatogenesis was limited compared to that of mGSC, this study developed a protocol by which $\mathrm{mESC}$ are induced into stable in vitro cell cultures with the mouse SSC ability to forerun spermatozoa and spermatids in adult mice testicles.

To date, most studies that focussed on targeted differentiation to generate male PGCLC in vitro have failed to showcase the expression of mature germ cell markers, which translates into PGCS in a very primary state. In 2018, Sosa et al. ${ }^{21}$ and colleagues derived PGCLC in vitro using Sasaki's two-step differentiation protocol that expressed pluripotency markers SOX17, TFAP2C, PRDM1, and OCT4 (Table 1)..$^{14,20-23}$ Once they were transplanted into previously sterilised mice of rhesus macaques testes, cells differentiated into a prespermatogonial phenotype, expressing VASA and MAGEA4. Furthermore, the expression of 5-hydroxymethylcytosine proved that the cells had commenced their epigenetic reprogramming. This study showed that physical contact between in vitro-induced PGCLC and the adult gonadal ridge is not required for them to differentiate. Nevertheless, both models of PGCLC transplantation halted the cell cycle and prevented their transition into spermatogonia. ${ }^{21}$

\section{Oogenesis}

Similar to the co-aggregate strategy employed by Zhou's group, Hikabe et al. ${ }^{24}$ used mice embryonic ovarian somatic cells to create reconstituted ovaries with $\mathrm{mPGCLC}$. By using cell culture protocols destined to mature primary follicles and mouse PGC into functional metaphase II oocytes,,25,26 the group obtained metaphase II oocytes that would later be fertilised via ICSI and produce healthy offspring, thus completing the full oogenesis in vitro. ${ }^{24,27}$ However, this method, had a live birth rate 20 times lower compared with oocytes in vivo attributable to errors during the epigenetic reprogramming caused by the short in vitro culture time to perform the in vitro differentiation compared to the time it lasts in vivo.
As previously described, the most recent methods for PGCLC maturation required the use of somatic cells, which makes the application of the protocol not appropriate for humans. Jung et al. ${ }^{22}$ tried to solve this by reproducing ovarian follicles in vitro without human gonad somatic cells as a supporting structure (Table 1).14,20-23 Starting hESC were differentiated into hPGC on top of fibroblast feeders cultured with BMP4 and BMP8A. Based on the knowledge that DAZL, a RNA-binding protein, regulates the transition from pluripotency of germ cells, this group overexpressed DAZL and BOULE in hPGCLC to induce meiosis in vitro. Once the expression of meiotic factors PRDM9 (expressed in the nucleus during preleptotene), H2AX (key histone for the remodelling of chromatin), and SYCP3 (which participates in the formation of the synaptonemal complex) was confirmed, the cells were transduced using human recombinant GDF4 and BMP15. After 9 days in culture, structures resembling follicles arose and the expression of ZP2 and NOBOX (oocyte markers) in the centre of the follicles was observed. Granulosa cells specific genes such as CYP19A and RSPO1 were also present. This was the first study that obtained a structure similar to an ovarian follicle that expressed both oocyte and granulosa cell factors. ${ }^{22}$

In summary, the in vitro differentiation of hPGCLC into functional gametes is still not completely successful since the functionality of the resulting cells is yet to be proven. A first approximation to this evaluation was performed by Yamashiro et al. $^{23}$ in 2018, who induced hPGCLC from a line of hiPSC and coaggregated them with somatic ovarian cells from 12-day-old mice embryos, thus creating xenogeneic reconstituted ovaries (Table 1)..$^{14,20-23}$ After 27 days of culture, both female and male cells derived from hPGCLC expressed early germ cell factors TFAP2C and SOX17, as well as DAZL and DDX 4 which determine that these had the potential to differentiate into gonocytes or oogonia. One month later, cells also expressed meiosis genes SYCP3 and REC8. The resulting cells formed a follicle-like structure and, from Day 120 of culture onwards, they expressed STRA8, indicating their readiness for meiosis. The authors also proved that the gene expression profile of these cells was very similar to that of oogonia in vivo. Furthermore, derived cells were able to respond to retinoic acid in preparation for meiosis ${ }^{23}$ and silenced male germline-specific 
genes. ${ }^{28}$ Finally, these cells progressively erased their paternal and maternal imprint; however, their reactivation of the inactive $X$ chromosome was proven to be inefficient. ${ }^{23}$ An additional summary of the most recent studies regarding the obtention of germ cells in vitro is shown in Table 1. ${ }^{14,20-23}$

\section{Transdifferentiation}

The birth of cellular reprogramming in 2006 allowed for the dedifferentiation of adult cells into pluripotent cells via the use of the Yamanaka factors. ${ }^{29,30}$ These iPSC could be later cultured with lineage-specific factors and, theoretically, differentiate into any other cell types. Based on this approach of reprogramming terminally differentiated cells into a different cellular lineage, Medrano et al. ${ }^{31}$ used a transdifferentiation strategy to obtain germ cells from somatic cells bypassing their pluripotent state. Starting from a foreskin fibroblast primary culture and a mesenchymal stem cell line, they overexpressed 6 key genes in the $X Y$ starting somatic cells: PRDM1, PRDM14, LIN28, DAZL, VASA, and SYCP3. The resulting cells exhibited an increase in expression of Fragilis and STELLA, markers for early germline differentiation, as well as early markers of PGC like SOX17. As the culture progressed, a decrease in the expression of premeiotic and early factors was observed, together with an increase in expression of late postmeiotic markers. However, the efficiency of the entire process was low: only $0.5-1.0 \%$ of the starting cells were able to complete meiosis. Once the epigenetics of these cells were analysed, the group observed that even though the starting cells were male $(n=46, X Y)$, the cells resulting from this genetic modification showed a significant loss of methylation in the paternal imprinting genes, while their methylation in the maternal imprinting genes increased, proving that in absence of a male gonad environment, the determination of female sex prevails. Finally, the group conducted a functional test in which in vitro obtained cells were able to colonise previously sterilised mouse testicles, even then complete spermatogenesis could not be completed because of interspecific differences between murine and human spermatogenesis. ${ }^{31}$ Despite achieving a low yield of recovered cells, this study became a starting point for protocols of direct differentiation of germ cells from adult somatic cells, which eliminates the need to isolate or induce pluripotent cells in order to achieve gametes.

In 2019, Zhang et al. ${ }^{32}$ reduced the number of transgenes required for the transdifferentiation of goat bone marrow stem cells (BMDSC) into spermatocyte precursor cells to 3 genes: STRA8, $B O U L E$, and $D A Z L .{ }^{33}$ The resulting cells were able to initiate meiosis and increase their expression of premeiotic factors STELLA and C-KIT compared to nontransfected cells, as well as postmeiotic markers PIWIL2 and SCYP3. Thus, the resulting cells were able to initiate meiosis, arrive to the point of recombination, and undergo epigenetic erasure of the imprinting genes. Nonetheless, they were unable to demonstrate that these cells completed meiosis to result in functional haploid spermatozoa. ${ }^{32}$

\section{ALTERNATIVES TO THE USE OF ESCS OR IPSCS}

Because of the inefficiency in the in vitro derivation of human gametes and the lack of evidence ensuring their safety and correct functionality, their use in a clinical setting for reproductive use is currently far from reality. Nevertheless, alternative stem cell sources exist to obtain germ cells for a more feasible shortterm clinical application.

SSC reside within the immature testicular tissue, which can be cryopreserved in prepubertal males that will be subjected to a potentially gonadotoxic treatment such as oncologic patients as a strategy to preserve their fertility. Following this strategy in 2019, Fayomi et al. ${ }^{34}$ reported the birth of the first nonhuman primate conceived using spermatozoa from rhesus macaque cryopreserved prepubertal testicular tissue which was later transplanted into the same castrated individual. In this study, one testicle was retrieved, cut into pieces, and cryopreserved. Immature tissue fragments were grafted under the skin from the same macaque's back and scrotum 7 months later. Once fragments were recovered and analysed 8-12 months post-grafting, seminiferous tubules exhibited complete spermatogenesis with multiple layers of VASA positive germ cells and acrosin positive post-meiotic spermatids. More than $70 \%$ of the seminiferous tubules contained elongated spermatogonia and/or spermatozoa. Spermatozoa were retrieved and 
microinjected into oocytes, and the resulting embryos displayed a $67 \%$ arrival to blastocyst rate and $25 \%$ gestation rate, comparable to rates obtained through natural ejaculation. Eleven blastocysts were transferred to six receptors and one successful birth was achieved. Although this experiment was performed in castrated animals, and therefore requires validation in sterile individuals with intact testicles, 34,35 it introduces the application of the in vivo maturation technique of cryopreserved fragments as a theoretically viable option for fertility preservation of prepubertal males. ${ }^{36}$

However, there are limitations to introducing this and other cellular transplantation techniques into clinical practice. Firstly, the origin of the transplanted sample presents the risk of reintroducing malignant cancer cells from the same patient. Thus, the auto-transplant strategy would not be applicable in children with leukaemia, lymphoma, or testicular cancer unless the safety of the transplant is ensured. ${ }^{34}$ Secondly, resumption of spermatogenesis in humans after reimplantation of immature testicular tissue has never been tried. Gonads are complex entities where cells require a specific environment to complete gametogenesis. Therefore, bioengineering approaches to this issue can be the creation of biocompatible scaffolds that mimic the testicle's or ovary's microenvironment to promote the differentiation of germinal stem cells into functional haploid gametes in vitro. ${ }^{37}$

Given the difficulty to retrieve gonadal somatic cells to act as a scaffold for PGC differentiation, several research groups looked for alternative sources of pluripotent stem cells to obtain germ cells. BMDSC can restore oogenesis by migrating and settling in the ovarian niche, facilitating follicular growth, neovascularisation, and proliferation of the ovarian stroma. This has been observed in mice ${ }^{38}$ and human patients with premature ovarian failure caused bychemotherapy treatment who, after a bone marrow transplant, became pregnant naturally. ${ }^{39}$ In fact, the ovarian infusion of autologous BMDSC in 17 low-responding women undergoing in vitro fertilisation cycles significantly increased the antral follicle count 2 weeks from the transplant, resulting in five live births. ${ }^{40}$ However, this technique does not involve the creation of new follicles or oocytes but mainly the 'awakening' of existing ones. ${ }^{41}$

\section{CURRENT CHALLENGES AND FUTURE PERSPECTIVES}

In humans, hPGCLC have been successfully induced from hESC and hiPSC; however, differentiation of these cells into mature and functional gametes is still ineffective. ${ }^{23}$ The available information about the development of the human germline is limited and focusses on the early stages of the differentiation process.

Besides its academic application, producing functional gametes in vitro would entail a substantial decrease in the shortage of ovum donors for fertility treatments and would offer a reproductive option to patients who are otherwise unable to have children. ${ }^{27}$ With respect to the latter, the current survival rate for childhood cancer is $80 \%$ and rising, which calls for the development of strategies that allow them to pursue their reproductive wish during adulthood whenever cryopreservation of mature gametes is not possible.,37 For this scenario, in males, there are several experimental techniques based on the cryopreservation of immature testicular tissue that are being studied in animal models; spermatogonial stem cell transplantation, ${ }^{35,42}$ testicular grafting, and in vitro spermatogenesis, which is currently nonviable with human cells since the process is arrested at the maturation stage of spermatogonia. The inefficiency of the latter is its greatest limitation, since most of the initial germ cells are lost during culture in the lab. 43,44

Furthermore, before gametes derived from hESC or hiPSC could be used for reproductive purposes, the genome and epigenome of both the starting cells and the resulting gametes need to be assessed to check that mutations or epimutations are not passed on to the offspring. ${ }^{6,45}$ It would be an irresponsibility for researchers to ignore the possibility of causing epigenetic errors that would result in aberrant embryogenesis or one that would entail unpredicted adverse effects in the offspring. ${ }^{46}$ Furthermore, since the last phase of the process has not been successfully reproduced with human cells in the laboratory, there is no proof of concept that spermatozoa generated from in vitro derivation of pluripotent stem cells would be able to correctly pack chromatin, substitute histones for protamines, and activate oocytes. Evidence that the female counterpart 
created using the same technique would be able to complete meiosis, repair DNA damage, disintegrate the nucleus of the spermatozoa, and form both pronuclei correctly would also be required. ${ }^{47}$

\section{CONCLUSION}

Overall, for now, the most promising use of in vitro gametogenesis is to serve as a study model, with a particular interest for researchers in basic biology, more than an alternative source of functional gametes. ${ }^{1,48}$ Nevertheless, recent advances described in this review suggest a promising future with a rise of new reproductive therapies following optimisation of these strategies.

\section{References}

1. Nagamatsu G, Hayashi K. Stem cells, in vitro gametogenesis and male fertility. Reproduction 2017;154(6):F79-91.

2. Sun $Y C$ et al. Reconstitution of gametogenesis in vitro: meiosis is the biggest obstacle. J Genet Genomics. 2014:41(3):87-95.

3. Tang WWW. Specification and epigenetic programming of the human germ line. Nat Rev Genet. 2016;17(10):585-600.

4. Chen D et al. The TFAP2C-regulated OCT4 naive enhancer is involved in human germline formation. Cell Rep. 2018:25(13):3591-602e5.

5. Kobayashi T, Surani MA. On the origin of the human germline. Development. 2018:145(16):dev150433.

6. Saitou M, Miyauchi H. Gametogenesis from pluripotent stem cells. Cell Stem Cell. 2016:18(6):721-35.

7. Ohinata $Y$ et al. A signaling principle for the specification of the germ cell lineage in mice. Cell. 2009;137(3):57184.

8. O'Brien MJ et al. A revised protocol for in vitro development of mouse oocytes from primordial follicles dramatically improves their developmental competence. Biol Reprod. 2003;68(5):1682-6.

9. Sato $T$ et al. In vitro production of fertile sperm from murine spermatogonial stem cell lines. Nat Commun. 2011;2:472.

10. Hayashi $\mathrm{K}$ et al. Reconstitution of the mouse germ cell specification pathway in culture by pluripotent stem cells. Cell. 2011;146(4):519-32.

11. Hackett JA et al. Tracing the transitions from pluripotency to germ cell fate with CRISPR screening. Nature Commun. 2018;9(1):4292.

12. Hayashi $\mathrm{K}$ et al. Offspring from oocytes derived from in vitro primordial germ cell-like cells in mice. Science. 2012:338(6109):971-5.

13. Irie $\mathrm{N}$ et al. SOX17 Is a critical specifier of human primordial germ cell fate.
Cell. 2015;160(1-2):253-68.

14. Sasaki $\mathrm{K}$ et al. Robust in vitro induction of human germ cell fate from pluripotent stem cells. Cell Stem Cell. 2015;17(2):178-94.

15. Nakaki $F$ et al. Induction of mouse germ-cell fate by transcription factors in vitro. Nature. 2013;501(7466):222-6.

16. Kobayashi $T$ et al. Principles of early human development and germ cell program from conserved model systems. Nature. 2017;546(7658):41620.

17. Murakami $\mathrm{K}$ et al. NANOG alone induces germ cells in primed epiblast in vitro by activation of enhancers. Nature. 2016:529(7586):403-7.

18. Fang $\mathrm{F}$ et al. A PAX5-OCT4-PRDM1 developmental switch specifies human primordial germ cells. Nat Cell Biol. 2018;20(6):655-65

19. Zhou $Q$ et al. Complete meiosis from embryonic stem cell-derived germ cells in vitro. Cell Stem Cell. 2016;18(3):330-40

20. Ishikura $Y$ et al. In vitro derivation and propagation of spermatogonial stem cell activity from mouse pluripotent stem cells. Cell Rep. 2016;17(10):2789804

21. Sosa $\mathrm{E}$ et al. Differentiation of primate primordial germ cell-like cells following transplantation into the adult gonadal niche. Nat Commun 2018;9(1):5339.

22. Jung $\mathrm{D}$ et al. In vitro differentiation of human embryonic stem cells into ovarian follicle-like cells. Nat Commun. 2017;8:15680.

23. Yamashiro $\mathrm{C}$ et al. Generation of human oogonia from induced pluripotent stem cells in vitro. Science. 2018;362(6412):356-60.

24. Hikabe $O$ et al. Reconstitution in vitro of the entire cycle of the mouse female germ line. Nature. 2016;539(7628):299-303

25. Morohaku $\mathrm{K}$ et al. Complete in vitro generation of fertile oocytes from mouse primordial germ cells. Proc Natl Acad Sci U S A. 2016:113(32):9021-6.

26. Hayashi K, Saitou M. Perspectives of germ cell development in vitro in mammals. Anim Sci J. 2014:85(6):61726

27. Makar K, Sasaki K. Roadmap of germline development and in vitro gametogenesis from pluripotent stem cells. Andrology. 2019;00:1-10.

28. Nagaoka Si, Saitou M. Reconstitution of female germ cell fate determination and meiotic initiation in mammals. Cold Spring Harb Symp Quant Biol. 2017;82:213-22.

29. Takahashi K, Yamanaka S. Induction of pluripotent stem cells from mouse embryonic and adult fibroblast cultures by defined factors. Cell. 2006;126(4):663-76.

30. Takahashi $\mathrm{K}$ et al. Induction of pluripotent stem cells from adult human fibroblasts by defined factors. Cell. 2007;131(5):861-72.

31. Medrano JV et al. Human Somatic cells subjected to genetic induction with six germ line-related factors display meiotic germ cell-like features. Sci Rep. 2016;6:24956.

32. Zhang $\mathrm{YL}$ et al. Induction of goat bone marrow mesenchymal stem cells into putative male germ cells using mRNA for STRA8, BOULE and DAZL. Cytotechnology. 2019;71(2):563-572.

33. Li PZ et al. Overexpression $\mathrm{ff}$ STRA8, BOULE, and DAZL genes promotes goat bone marrowderived mesenchymal stem cells in vitro transdifferentiation toward putative male germ cells. Reprod Sci. 2017:24(2):300-12

34. Fayomi $\mathrm{AP}$ et al. Autologous grafting of cryopreserved prepubertal rhesus testis produces sperm and offspring. Science. 2019;363(6433):1314-9.

35. Pelzman DL et al. Progress in translational reproductive science: testicular tissue transplantation and in vitro spermatogenesis. Fertil Steril. 2020;113(3):500-9 
36. Focus on Reproduction. More developments in in vitro gametogenesis. 2019. Available at: https://www.focusonreproduction. eu/article/ESHRE-MeetingsMore-developments-in-in-vitrogametogenesis. Last accessed: 30 March 2019.

37. Giudice MG et al. Update on fertility restoration from prepubertal spermatogonial stem cells: how far are we from clinical practice? Stem Cell Res. 2017;21:171-7.

38. Martin JJ et al. Reconstitution of oogenesis in mice using bone marrow-derived stem cells aggregated with fetal ovarian somatic cells. Cell. 2019;8(2):93.

39. Herraiz $\mathrm{S}$ et al. Fertility rescue and ovarian follicle growth promotion by bone marrow stem cell infusion. Fertil
Steril. 2018;109(5):908-18e2.

40. Herraiz S et al. Autologous stem cel ovarian transplantation to increase reproductive potential in patients who are poor responders. Fertil Steril. 2018;110(3):496-505.e1.

41. Bhartiya D et al. Making gametes from alternate sources of stem cells: past, present and future. Reprod Biol Endocrinol. 2017:15(1):89.

42. Tian $\mathrm{J}$ et al. Relative safety of various spermatogenic stem cell purification methods for application in spermatogenic stem cell transplantation. Stem Cell Res Ther. 2019;10(1):382.

43. Medrano JV et al. [Current state regarding fertility cryopreservation in pre-pubertal boys]. Rev Int Androl. 2018;18(1):27-34. (In Spanish).
44. Medrano JV et al. Basic and clinical approaches for fertility preservation and restoration in cancer patients. Trends Biotechnol. 2018;36(2):199215.

45. Mathews DJ et al. Pluripotent stem cell-derived gametes: truth and (potential) consequences. Cell Stem Cell. 2009;5(1):11-4

46. Ishii T, Saitou M. Promoting in vitro gametogenesis research with a social understanding. Trends Mol Med. 2017;23(11):985-8.

47. Handel Ma et al. Applying "gold standards" to in-vitro-derived germ cells. Cell. 2014;157(6):1257-61.

48. Ge W et al. Skin-derived stem cells as a source of primordial germ celland oocyte-like cells. Cell Death Dis. 2016;7(11):e2471. 